\title{
A scalable, fully implicit algorithm for the reduced two-field low- $\beta$ extended MHD model
}

\author{
L. Chacón ${ }^{\mathrm{a}, *}$, A. Stanier ${ }^{\mathrm{a}}$ \\ ${ }^{a}$ Los Alamos National Laboratory, Los Alamos, NM 87545
}

\begin{abstract}
We demonstrate a scalable fully implicit algorithm for the two-field low- $\beta$ extended MHD model. This reduced model describes plasma behavior in the presence of strong guide fields, and is of significant practical impact both in nature and in laboratory plasmas. The model displays strong hyperbolic behavior, as manifested by the presence of fast dispersive waves, which make a fully implicit treatment very challenging. In this study, we employ a Jacobian-free Newton-Krylov nonlinear solver, for which we propose a physics-based preconditioner that renders the linearized set of equations suitable for inversion with multigrid methods. As a result, the algorithm is shown to scale both algorithmically (i.e., the iteration count is insensitive to grid refinement and timestep size) and in parallel in a weak-scaling sense, with the wall-clock time scaling weakly with the number of cores for up to 4096 cores. For a $4096 \times 4096$ mesh, we demonstrate a wall-clock-time speedup of four orders of magnitude with respect to explicit algorithms. The model is validated linearly (against linear theory predictions) and nonlinearly (against fully kinetic simulations), demonstrating excellent agreement.
\end{abstract}

Keywords: implicit algorithms, extended MHD, JFNK, physics-based preconditioning, parallel multigrid PACS:

\section{Introduction}

The low- $\beta$ reduced extended magnetohydrodynamics (two-field) model is obtained by taking the largeguide-field and cold-ion limit [1, 2, 3] of the extended MHD model [4]. The resulting model is appealing owing to its simplicity, and because it retains key two-fluid physics that can be important in a wide range of laboratory magnetic confinement devices [5, 6, 7, 8], the solar corona [9], and other astrophysical plasmas in which large guide fields are present. One important application is Tokamak magnetically-confined plasmas such as ITER.

The relevance of the two-fluid system in the study of magnetized plasmas is evidenced by the large body of literature discussing it $[10,11,2,12,13,14,15,16,17,18,19,20]$. However, the numerical integration of this system is non-trivial because it supports disparate time and length scales, which demand both spatial adaptivity and efficient implicit integration methods for efficiency. The large time-scale disparity originates in the presence of fast dispersive waves [21], which result in significant numerical stiffness and the need of high-order dissipation operators to prevent numerical noise in the nonlinear regime [22, 23]. Both fast dispersive waves and high-order dissipation stress numerical algorithms, and benefit from an implicit treatment.

\footnotetext{
${ }^{*}$ Corresponding author

Email address: chacon@lanl.gov (L. Chacón)
} 
Despite its physics relevance, to our knowledge there is scant effort devoted towards the development of modern, efficient implicit algorithms for the numerical solution of the two-field model, with many practitioners relying on explicit methods [24, 25]. The MRC code [26] integrates several extended MHD systems, including the one of interest here. It employs nonlinear implicit time-stepping iterated with Newton methods, but inverts the Jacobian using direct solvers. A Newton-Krylov-Schwarz implicit parallel solver is proposed in Ref. [27] for the two-field model. Within each processor patch, the algorithm employs incomplete ILU methods with various degrees of overlap. For a time step of $\Delta t=1 \tau_{A}$ and a $1980 \times 1980$ mesh, the solver is reported to require between 10 and 25 nonlinear iterations per time step, and between 40 and 250 linear iterations per nonlinear iteration, depending on the point in time in the simulation and the number of processors employed. The algorithm is demonstrated to scale reasonably up to 2025 processors (in a strong-scaling sense on a fixed $1980 \times 1980$ mesh), but only after careful optimization of the domain overlap. Reported speedups with respect to explicit approaches are at most of an order of magnitude. More recently, Ref. [28] proposes a variational discretization for the collisionless reduced two-fluid XMHD model considering only electron inertia that exactly conserves energy and magnetic helicity. The approach is implicit, employing Jacobian-free Newton-Krylov (JFNK) solvers and preconditioned using an extension of the preconditioners proposed in [29] for resistive reduced MHD to the electron-inertia case. The study focused on the derivation of the discretization, with minimal attention paid to parallelization or performance.

The focus of this paper is to demonstrate an efficient, optimal nonlinearly implicit parallel algorithm for the two-field model. The approach uses JFNK methods, effectively preconditioned using physics-based approximations of the Jacobian system that are multigrid-friendly, and therefore deliver optimal convergence rates. The preconditioning approach presented here leverages earlier developments of effective physicsbased preconditioners for MHD [29, 30] and extended MHD [31, 32], and in particular employs a similar parabolization strategy to that presented in these studies. However, the unique features of the two-field algorithm presented here, together with its breadth of applications, demand careful documentation. We have implemented the algorithm in the PIXIE2D nonlinear reduced MHD solver framework [29, 31]. We demonstrate by numerical experiment that our algorithm features logarithmic parallel weak scaling, and for the same time step and comparable mesh resolutions as in Ref. [27], it converges in a handful of linear and nonlinear iterations (typically less than 5) and provides speedup with respect to explicit methods of many orders of magnitude ( 4 orders of magnitude for the largest mesh considered in this study, $4096 \times 4096$ ).

The paper is organized as follows. Section 2 introduces the two-field model, and its salient features that challenge its algorithmic treatment. Section 3 briefly introduces JFNK nonlinear solvers and preconditioning. The physics-based preconditioner for the two-field system is developed in Sec. 4. We present linear and nonlinear verification results, along with serial and parallel performance results in Sec. 5. Finally, we conclude in Sec. 6.

\section{Formulation and properties of the two-field low- $\beta$ extended MHD model}

We develop an efficient preconditioning strategy for the so-called two-field extended MHD model, valid in the low- $\beta$, cold-ion regime $[2,3,14]$, and given by:

$$
\begin{aligned}
\partial_{t} F+\mathbf{v} \cdot \nabla F+\rho_{s}^{2} \mathbf{B} \cdot \nabla \Omega & =\eta \nabla^{2} \psi-\eta_{H} \nabla^{4} \psi \\
\partial_{t} \Omega+\mathbf{v} \cdot \nabla \Omega-\mathbf{B} \cdot \nabla J & =\mu \nabla^{2} \Omega .
\end{aligned}
$$

The model assumes constant temperature. In these equations, $F=\psi-d_{e}^{2} J$ is the conserved Casimir invariant in the dissipationless limit, $\psi$ is the poloidal flux, $J=\nabla^{2} \psi$ is the parallel current, $\Omega=\nabla^{2} \phi$ is the vorticity, $\phi$ the streamfunction, $\mathbf{v}=\mathbf{z} \times \nabla \phi$ is the flow velocity, $\mathbf{z}$ is the unit vector in the direction of the guide field $B_{z}$, and $\mathbf{B}=\mathbf{B}_{p}+B_{z} \mathbf{z}$ is the magnetic field, with $\mathbf{B}_{p}=\mathbf{z} \times \nabla \psi$ the poloidal magnetic field. Quantities have been normalized using Alfvén units (Alfvén velocity $v_{A}=B_{0} / \sqrt{\mu_{0} m_{i} n_{0}}$ and length $L$, with $B_{0}, n_{0}$ 
the reference magnetic field and density, respectively, $m_{i}$ the ion mass, and $\mu_{0}$ the vacuum permeability), with $\eta$ the normalized resistivity, $\mu$ the normalized viscosity, $\eta_{H}$ the normalized electron viscosity (also termed hyperresistivity), $d_{e}=c / \omega_{p e} L$ the normalized electron skin depth (with $c$ the speed of light and $\omega_{p e}$ the electron plasma frequency), and $\rho_{s}=\sqrt{T_{e} / m_{i}} / \Omega_{i} L$ (with $T_{e}$ the electron temperature and $\Omega_{i}$ the ion gyrofrequency) the normalized sound Larmor radius. The two-fluid parameters $d_{e}$ and $\rho_{s}$ are taken as constant and uniform. For the following, we also define the velocity $\mathbf{v}_{s}=\mathbf{z} \times \nabla\left(\phi-\rho_{s}^{2} \Omega\right)$.

Equations 1 and 2 can support fast dispersive waves with dispersion relation $\omega \sim k_{\|} k$, with $\omega$ the frequency and $k$ the wavenumber. This can be readily seen by linearizing them for a homogeneous plasma in a uniform magnetic field $\left(\phi_{0}=\Omega_{0}=0, \mathbf{B}_{p}=\mathbf{B}_{p, 0},\left|\mathbf{B}_{p, 0}\right|=1, J_{0}=0, F_{0}=\psi_{0}=x\right)$, to find in the collisionless limit:

$$
\begin{aligned}
\partial_{t} \tilde{F}-\mathbf{B}_{0} \cdot \nabla\left(\tilde{\phi}-\rho_{s}^{2} \tilde{\Omega}\right) & =0, \\
\partial_{t} \tilde{\Omega}-\mathbf{B}_{0} \cdot \nabla\left(\nabla^{2} \tilde{\psi}\right) & =0 .
\end{aligned}
$$

Employing the Fourier ansatz $\tilde{\chi}=\hat{\chi} e^{i(\omega t-\mathbf{k} \cdot \mathbf{x})}$, and denoting $k_{\|}=\mathbf{B}_{0} \cdot \mathbf{k}$, we find:

$$
\left[\begin{array}{cc}
\omega\left(1+d_{e}^{2} k^{2}\right) & -k_{\|}\left(1+\rho_{s}^{2} k^{2}\right) \\
-k_{\|} & \omega
\end{array}\right]\left(\begin{array}{c}
\tilde{\psi} \\
\tilde{\phi}
\end{array}\right)=\left(\begin{array}{l}
0 \\
0
\end{array}\right)
$$

The vanishing determinant yields the dispersion relation:

$$
\omega^{2}=k_{\|}^{2} \frac{1+\rho_{s}^{2} k^{2}}{1+d_{e}^{2} k^{2}}
$$

which only gives fast dispersive waves for $\rho_{s}>d_{e}$ [or $\beta_{e} / 2>m_{e} / m_{i}$, with $\beta_{e}=2 \mu_{0} p_{e} / B^{2}$ the electron $\beta$ parameter] [21]. The dispersion relation yields the Alfvén wave $\left(\omega \approx \pm k_{\|}\right)$for either $\rho_{s}=d_{e}$ or $k^{2} \rho_{s}^{2}, k^{2} d_{e}^{2} \ll 1$, the fast dispersive kinetic Alfvén wave $\left(\omega \approx \pm \rho_{s} k_{\|} k\right)$ for $k \rho_{s}>1>k d_{e}$, and a scaled Alfvén wave $\left(\omega \approx \pm \frac{\rho_{s}}{d_{e}} k_{\|}\right)$for $k^{2} \rho_{s}^{2}, k^{2} d_{e}^{2} \gg 1$. Fast dispersive waves introduce significant numerical difficulty for the implicit integration of the low- $\beta$ extended MHD model. For this task, we consider preconditioned Jacobian-free Newton-Krylov methods.

Equations 1 and 2 are discretized in a similar fashion as described in Refs. [29, 31], using secondorder finite differences in space, and a $\theta$-scheme (Crank-Nicolson for $\theta=1 / 2$ ) or a second-order Backward Differentiation formula (BDF2 [33]) in time. Boundary conditions are either periodic, or perfect-conductor and impenetrable-wall. One difference between our current implementation and that in the references is that we do not consider the streamfunction equation, $\Omega=\nabla^{2} \phi$, as an independent equation in the residual. Instead, we reformulate Eq. 2 in terms of the streamfunction $\phi$ as:

$$
\partial_{t} \phi+\nabla^{-2}(\mathbf{v} \cdot \nabla \Omega-\mathbf{B} \cdot \nabla J)=\mu \nabla^{2} \phi .
$$

This formulation requires a Poisson inversion per residual evaluation, and is therefore typically more computationally intensive than the one in Eqs. 1, 2. Nevertheless, we have found Eq. 6 to be much better behaved for large problem sizes, for which the vorticity equation (Eq. 2) suffers from noise originating in the third-order $\mathbf{B} \cdot \nabla\left(\nabla^{2} \psi\right)$ operator (which is the highest-order differential operator in the vorticity equation), whereas Eq. 6 does not (since $\nabla^{-2}\left(\mathbf{B} \cdot \nabla \nabla^{2} \psi\right)$ is formally only first-order). The required Poisson inversion in the residual is converged to a relative tolerance one order of magnitude smaller than the relative nonlinear tolerance of the Newton-Krylov nonlinear solver (see the next section), but does not spoil the optimality of our algorithm, as we shall demonstrate later in this study. 


\section{Jacobian-free Newton-Krylov iterative solver}

We provide here a brief introduction to Jacobian-free Newton-Krylov methods (JFNK). The motivated reader can find extensive discussions on this approach elsewhere [34]. Newton's method solves the nonlinear system $\mathbf{G}(\mathbf{x})=\mathbf{0}$ iteratively by inverting linear systems of the form:

$$
\left.\frac{\partial \mathbf{G}}{\partial \mathbf{x}}\right|_{k} \delta \mathbf{x}_{k}=-\mathbf{G}\left(\mathbf{x}_{k}\right),
$$

with $\mathbf{x}_{k+1}=\mathbf{x}_{k}+\delta \mathbf{x}_{k}$. Nonlinear convergence is achieved when:

$$
\left\|\mathbf{G}\left(\mathbf{x}_{k}\right)\right\|_{2}<\varepsilon_{a}+\varepsilon_{r}\left\|\mathbf{G}\left(\mathbf{x}_{0}\right)\right\|_{2}=\varepsilon_{t},
$$

where $\|\cdot\|_{2}$ is the $L_{2}$-norm (euclidean norm), $\varepsilon_{a}=\sqrt{N} \times 10^{-15}$ (with $N$ the total number of degrees of freedom) is an absolute tolerance based on machine round-off error for double precision to avoid converging below round-off, $\varepsilon_{r}$ is the Newton relative convergence tolerance (set to $10^{-4}$ in this work), and $\mathbf{G}\left(\mathbf{x}_{0}\right)$ is the initial residual.

Such linear systems are solved iteratively with Krylov methods, which only require matrix-vector products to proceed. Because the linear system matrix is a Jacobian matrix, such matrix-vector products can be implemented Jacobian-free using the Gateaux derivative:

$$
\left.\frac{\partial \mathbf{G}}{\partial \mathbf{x}}\right|_{k} \mathbf{v}=\lim _{\varepsilon \rightarrow 0} \frac{\mathbf{G}\left(\mathbf{x}_{k}+\varepsilon \mathbf{v}\right)-\mathbf{G}\left(\mathbf{x}_{k}\right)}{\varepsilon},
$$

where in practice a small but finite $\varepsilon$ is employed (p. 79 in [34]). Thus, the evaluation of the Jacobian-vector product only requires the function evaluation $\mathbf{G}\left(\mathbf{x}_{k}+\varepsilon \mathbf{v}\right)$, and there is no need to form or store the Jacobian matrix. This, in turn, allows for a memory-efficient implementation.

An inexact Newton method [35] is used to adjust the convergence tolerance of the Krylov method at every Newton iteration according to the size of the current Newton residual, as follows:

$$
\left\|J_{k} \delta \mathbf{x}_{k}+\mathbf{G}\left(\mathbf{x}_{k}\right)\right\|_{2}<\zeta_{k}\left\|\mathbf{G}\left(\mathbf{x}_{k}\right)\right\|_{2}
$$

where $\zeta_{k}$ is the inexact Newton parameter and $J_{k}=\left.\frac{\partial \mathbf{G}}{\partial \mathbf{x}}\right|_{k}$ is the Jacobian matrix. Thus, the convergence tolerance of the Krylov method is loose when the Newton state vector $\mathbf{x}_{k}$ is far from the nonlinear solution, but tightens as $\mathbf{x}_{k}$ approaches the solution. Hence, the linear solver works the hardest when the Newton state vector is closest to the nonlinear root. Superlinear convergence rates of the inexact Newton method are possible if the sequence of $\zeta_{k}$ is chosen properly (p. 105 in [34]). Here, we employ the same prescription as in Ref. [31]:

$$
\begin{aligned}
\zeta_{k}^{A} & =\gamma\left(\frac{\left\|\mathbf{G}\left(\mathbf{x}_{k}\right)\right\|_{2}}{\left\|\mathbf{G}\left(\mathbf{x}_{k-1}\right)\right\|_{2}}\right)^{\alpha} \\
\zeta_{k}^{B} & =\min \left[\zeta_{\max }, \max \left(\zeta_{k}^{A}, \gamma \zeta_{k-1}^{\alpha}\right)\right] \\
\zeta_{k} & =\min \left[\zeta_{\max }, \max \left(\zeta_{k}^{B}, \gamma \frac{\varepsilon_{t}}{\left\|\mathbf{G}\left(\mathbf{x}_{k}\right)\right\|_{2}}\right)\right]
\end{aligned}
$$

with $\alpha=1.5, \gamma=0.9$, and $\zeta_{\max }=0.8$. The convergence tolerance $\varepsilon_{t}$ is defined in Eq. 7 . In this prescription, the first step ensures superlinear convergence (for $\alpha>1$ ), the second avoids volatile decreases in $\zeta_{k}$, and the last avoids oversolving in the last Newton iteration.

A further advantage of Krylov methods is that they can be preconditioned by considering the alternate (but equivalent) systems $J_{k} P_{k}^{-1} P_{k} \delta \mathbf{x}_{k}=-\mathbf{G}_{k}$ (right preconditioning) or $P_{k}^{-1} J_{k} \delta \mathbf{x}_{k}=-P_{k}^{-1} \mathbf{G}_{k}$ (left preconditioning). Such preconditioned systems can be straightforwardly and efficiently implemented in the Krylov 
algorithm as two consecutive matrix-vector products. A crucial feature of preconditioning is that, while it can substantially improve the convergence properties of the Krylov iteration if $P_{k}^{-1} \approx J_{k}^{-1}$, it does not alter the solution of the Jacobian system upon convergence (because the solution $\delta \mathbf{x}_{k}$ of the preconditioned system is the same as that of the original system). Therefore, one can explore suitable approximations in the preconditioner for efficiency purposes without compromising the accuracy of the converged result.

This paper concentrates on the development of a scalable, robust preconditioning strategy for the NewtonKrylov inversion of the low- $\beta$ extended MHD model outlined in Eqs. 1-2. Right preconditioning is favored here, because the right-hand-side of the Jacobian system $-\mathbf{G}_{k}$ (which measures nonlinear convergence) is unaffected by the preconditioner. In practice, right preconditioning is implemented in two steps, namely, a linear solve $J_{k} P_{k}^{-1} \delta \mathbf{y}_{k}=-\mathbf{G}_{k}$, and a posteriori application of the preconditioner to find $\delta \mathbf{x}_{k}=P_{k}^{-1} \delta \mathbf{y}_{k}$. We use FGMRES [36] as the Krylov method of choice for its robustness in non-symmetric, indefinite systems (which is the case here) and the added flexibility in the preconditioning stage. We discuss our approach to preconditioning next.

\section{Physics-based preconditioning strategy}

We begin the development of the physics-based preconditioner with a linearized version of Eqs. 1 and 6 (after applying a Laplacian operator) about arbitrary states $\phi_{0}, \psi_{0}$, and $\Omega_{0}$, to find:

$$
\begin{aligned}
\mathscr{L}_{\eta}^{s} \tilde{\psi}-\mathbf{B}_{0}^{*} \cdot \nabla \tilde{\phi}+\rho_{s}^{2} \mathbf{B}_{0} \cdot \nabla \tilde{\Omega} & =-\mathbf{G}_{\psi}, \\
\mathscr{L}_{\mu} \tilde{\Omega}+\tilde{\mathbf{v}} \cdot \nabla \Omega_{0}-\mathbf{B}_{0} \cdot \nabla\left(\nabla^{2} \tilde{\psi}\right)+\left(\mathbf{z} \times \nabla J_{0}\right) \cdot \nabla \tilde{\psi} & =-\nabla^{2} \mathbf{G}_{\phi},
\end{aligned}
$$

with $\tilde{\Omega}=\nabla^{2} \tilde{\phi}$. Here, $\mathbf{G}_{\psi}$ and $\mathbf{G}_{\phi}$ are the residuals for the $\psi$ and $\phi$ equations, respectively. In these equations, $\mathbf{B}_{0}^{*}=\mathbf{z} \times \nabla F_{0}$, and the operators $\mathscr{L}$ are advection-diffusion operators, of the form:

$$
\begin{aligned}
& \mathscr{L}_{\eta}^{s}=\frac{\mathbb{I}-d_{e}^{2} \nabla^{2}}{\Delta t}+\mathbf{v}_{s, 0} \cdot \nabla-d_{e}^{2}\left(\mathbf{v}_{0} \cdot \nabla\right) \nabla^{2}-\eta \nabla^{2}+\eta_{H} \nabla^{4}, \\
& \mathscr{L}_{\mu}=\frac{\mathbb{I}}{\Delta t}+\mathbf{v}_{0} \cdot \nabla-\mu \nabla^{2} .
\end{aligned}
$$

Following Ref. [29], and critical to the derivation of the preconditioner, we approximate the left hand side of Eq. 11 as:

$$
\mathscr{L}_{\mu} \tilde{\Omega}+\tilde{\mathbf{v}} \cdot \nabla \Omega_{0}-\mathbf{B}_{0} \cdot \nabla\left(\nabla^{2} \tilde{\psi}\right)+\left(\mathbf{z} \times \nabla J_{0}\right) \cdot \nabla \tilde{\psi} \approx \nabla^{2}\left[\mathscr{L}_{\mu} \tilde{\phi}-\mathbf{B}_{0} \cdot \nabla \tilde{\psi}\right],
$$

which allows us to write it as:

$$
\mathscr{L}_{\mu} \tilde{\phi}-\mathbf{B}_{0} \cdot \nabla \tilde{\psi}=-\mathbf{G}_{\phi}
$$

Solving for $\tilde{\phi}$ :

$$
\tilde{\phi}=\mathscr{L}_{\mu}^{-1}\left[\mathbf{B}_{0} \cdot \nabla \tilde{\psi}-\mathbf{G}_{\phi}\right],
$$

and replacing this equation and Eq. 11 (neglecting the $\tilde{\mathbf{v}} \cdot \nabla \Omega_{0}$ term and solving for $\tilde{\Omega}$ ) into Eq. 10 , we find an equation for $\tilde{\psi}$ that encodes all fast-wave temporal scales:

$$
\begin{aligned}
\mathscr{L}_{\eta}^{s} \tilde{\psi}-\left(\mathbf{B}_{0}^{*} \cdot \nabla\right) \mathscr{L}_{\mu}^{-1}\left(\mathbf{B}_{0} \cdot \nabla\right) \tilde{\psi} & -\rho_{s}^{2}\left(\mathbf{B}_{0} \cdot \nabla\right) \mathscr{L}_{\mu}^{-1}\left(\mathbf{z} \times \nabla J_{0}\right) \cdot \nabla \tilde{\psi}+\rho_{s}^{2}\left(\mathbf{B}_{0} \cdot \nabla\right) \mathscr{L}_{\mu}^{-1}\left(\mathbf{B}_{0} \cdot \nabla\right) \nabla^{2} \tilde{\psi} \\
& \approx-\mathbf{G}_{\psi}+\left(\mathbf{B}_{0}^{*} \cdot \nabla\right) \mathscr{L}_{\mu}^{-1}\left[-\mathbf{G}_{\phi}\right]-\rho_{s}^{2}\left(\mathbf{B}_{0} \cdot \nabla\right) \mathscr{L}_{\mu}^{-1} \nabla^{2}\left[-\mathbf{G}_{\phi}\right] .
\end{aligned}
$$

Equation 13 has effectively combined the hyperbolic couplings present in Eqs. 10-11 (similarly to earlier studies [29, 31, 30]), which now appear as second- and fourth-order anisotropic diffusion operators, corresponding to linear and dispersive wave propagation, respectively. As written, however, this equation is impractical to solve due to the presence of $\mathscr{L}_{\mu}^{-1}$ operators. We get around this difficulty by postulating a 
stationary iterative procedure (as was done in Ref. [29]) based on a diagonal splitting of the operator $\mathscr{L}_{\mu}$ in both Eqs. 11 and 12, i.e.:

$$
\mathscr{L}_{\mu} \tilde{\phi} \approx \mathscr{D}_{\mu} \tilde{\phi}^{m+1}-\left(\mathscr{D}_{\mu}-\mathscr{L}_{\mu}\right) \tilde{\phi}^{m}
$$

The index $m$ corresponds to the stationary iteration level. Solving for $\tilde{\phi}^{m+1}$ and $\tilde{\Omega}^{m+1}$ in Eqs. 11 and 12 gives:

$$
\begin{aligned}
\tilde{\phi}^{m+1} & =\left(\mathbb{I}-\mathscr{D}_{\mu}^{-1} \mathscr{L}_{\mu}\right) \tilde{\phi}^{m}+\mathscr{D}_{\mu}^{-1}\left[\mathbf{B}_{0} \cdot \nabla \tilde{\psi}^{m+1}-\mathbf{G}_{\phi}\right]=\mathscr{D}_{\mu}^{-1} \mathbf{B}_{0} \cdot \nabla \tilde{\psi}^{m+1}+\mathrm{rhs}_{\phi}^{m}, \\
\tilde{\Omega}^{m+1} & =\left(\mathbb{I}-\mathscr{D}_{\mu}^{-1} \mathscr{L}_{\mu}\right) \tilde{\Omega}^{m}+\mathscr{D}_{\mu}^{-1}\left[-\tilde{\mathbf{v}}^{m} \cdot \nabla \Omega_{0}+\mathbf{B}_{0} \cdot \nabla\left(\nabla^{2} \tilde{\psi}^{m+1}\right)+\tilde{\mathbf{B}}^{m+1} \cdot \nabla J_{0}+\nabla^{2}\left[-\mathbf{G}_{\phi}\right]\right] \\
& =\mathscr{D}_{\mu}^{-1}\left[\mathbf{B}_{0} \cdot \nabla\left(\nabla^{2} \tilde{\psi}^{m+1}\right)-\left(\mathbf{z} \times \nabla J_{0}\right) \cdot \nabla \tilde{\psi}^{m+1}\right]+\operatorname{rhs}_{\Omega}^{m}
\end{aligned}
$$

Here:

$$
\begin{aligned}
\operatorname{rhs}_{\phi}^{m} & =\left(\mathbb{I}-\mathscr{D}_{\mu}^{-1} \mathscr{L}_{\mu}\right) \tilde{\phi}^{m}+\mathscr{D}_{\mu}^{-1}\left[-\mathbf{G}_{\phi}\right] \\
\operatorname{rhs}_{\Omega}^{m} & =\left(\mathbb{I}-\mathscr{D}_{\mu}^{-1} \mathscr{L}_{\mu}\right) \tilde{\Omega}^{m}+\mathscr{D}_{\mu}^{-1}\left[\tilde{\mathbf{v}}^{m} \cdot \nabla \Omega_{0}+\nabla^{2}\left[-\mathbf{G}_{\phi}\right]\right]
\end{aligned}
$$

Replacing these results into Eq. 10 finally gives a tractable equation for $\tilde{\psi}$ :

$$
P_{S I} \tilde{\psi}^{m+1}=-\mathbf{G}_{\psi}+\left(\mathbf{B}_{0}^{*} \cdot \nabla\right)\left[\mathrm{rhs}_{\phi}^{m}\right]-\rho_{s}^{2}\left(\mathbf{B}_{0} \cdot \nabla\right)\left[\mathrm{rhs}_{\Omega}^{m}\right] .
$$

with:

$$
\begin{aligned}
P_{S I} \tilde{\psi}^{m+1} & =\mathscr{L}_{\eta}^{s} \tilde{\psi}^{m+1}-\left(\mathbf{B}_{0}^{*} \cdot \nabla\right) \mathscr{D}_{\mu}^{-1}\left(\mathbf{B}_{0} \cdot \nabla\right) \tilde{\psi}^{m+1} \\
& +\rho_{s}^{2}\left(\mathbf{B}_{0} \cdot \nabla\right) \mathscr{D}_{\mu}^{-1}\left[\left(\mathbf{B}_{0} \cdot \nabla\right) \nabla^{2} \tilde{\psi}^{m+1}-\left(\mathbf{z} \times \nabla J_{0}\right) \cdot \nabla \tilde{\psi}^{m+1}\right] .
\end{aligned}
$$

Equation 16, iterated together with Eqs. 14 and 15, comprises the proposed physics-based preconditioner. After the iteration, we clean some of the approximations introduced by resolving $\tilde{\Omega}$ from Eq. 11 (using $\tilde{\Omega}^{m+1}$ as an initial guess), and $\tilde{\phi}$ from $\nabla^{2} \tilde{\phi}=\tilde{\Omega}$ (also using $\tilde{\phi}^{m+1}$ as an initial guess). It is conceptually similar to the one for the high- $\beta$ two-fluid MHD model derived in Ref. [31], and the approximate multigrid inversion methods proposed there can be readily applied in this context.

\section{Numerical results}

In what follows, we present numerical examples that demonstrate the correctness of our implementation (verification), and we characterize the efficiency of the proposed preconditioning strategy. For verification, we employ both linear examples (tearing mode instability) and nonlinear examples (by comparing against kinetic simulations in regimes of interest). We characterize the efficiency and algorithmic scalability properties of the approach by performing grid and time-step convergence studies.

For the residual Poisson solve in the streamfunction equation, we use multigrid-preconditioned CG, converged to a tolerance of $0.1 \varepsilon_{r}=10^{-5}$. We employ classical fully parallel multigrid (MG) methods to approximate $P_{S I}^{-1}$ and the inverse Laplacian operators in the preconditioner, and to precondition the Poisson solve in the residual evaluation. We employ agglomeration for the restriction step, and first-order prolongation. As a smoother, we employ three passes of damped Jacobi (p. 10 in [37]; p. 118 in [38]), with weight $\omega=0.7$, for both the restriction and the prolongation steps. Unless otherwise specified, we employ the smoother as the coarse grid solver as well. In MG jargon, such V-cycle is identified as V(3,3), where the two integers indicate restriction and prolongation smoothing steps, respectively. For the linear solves in the preconditioner, we use the lesser of either $2 \mathrm{~V}(3,3)$ multigrid cycles or a convergence tolerance of $10^{-1}$.

The explicit solver employed in this work as a reference to calculate implicit CPU speedup is a secondorder Runge-Kutta method, which requires two function evaluations per time step. Each step requires 
inversion of elliptic couplings $\left(\nabla^{2} \phi=\Omega\right.$ and $\left.\left(1 / \Delta t-d_{e}^{2} \nabla^{2}\right) \Psi=F\right)$, which we solve using multigridpreconditioned CG to same relative tolerance as the implicit solve $\left(\varepsilon_{r}=10^{-4}\right)$. This explicit algorithm is used as a practical measure of numerical stiffness, and is a natural benchmark of algorithmic performance owing to its simplicity.

\subsection{Linear verification: doubly periodic tearing mode}

We consider a cosine doubly periodic tearing mode (DPTM) equilibrium [11, 12, 15, 27], given by:

$$
\psi_{0}=J_{0}=\cos (y)
$$

in a doubly periodic domain of dimensions $x \in(-2 \pi, 2 \pi)$ and $y \in(-\pi, \pi)$ (which is unstable to the tearing mode with wavenumber $\left.k_{x}=\frac{2 \pi}{L_{x}}=0.5\right)$. We choose the following parameters: $\rho_{s}=0.1, d_{e}=0.01, \eta=\mu=$ $10^{-5}, \eta_{H}=0$ (sound-radius dominated, case I), and $\rho_{s}=0.01, d_{e}=0.1, \eta=\mu=10^{-5}, \eta_{H}=0$ (electroninertia dominated, case II). For both cases, we consider a $128 \times 256$ grid, uniform in the $x$-direction and packed in the $y$-direction at the rational surfaces $(y=0, \pi)$, at which $\Delta y=2 \times 10^{-3}$, and increasing away from them according to a cosine map $y=\xi+a \cos (\xi)$, with $y$ the physical coordinate and $\xi$ the logical one.

We have computed the linear eigenvalues for these parameters using an independent linear eigenvalue solver, and obtained a real tearing growth rate of $\gamma=2.5797 \times 10^{-3}$ (case I) and $\gamma=5.2841 \times 10^{-3}$ (case II). Our nonlinear solver finds $\gamma=2.5773 \times 10^{-3}$ and $\gamma=5.3036 \times 10^{-3}$, respectively, i.e., with errors of only a few parts in $10^{3}$. The eigenvalues from the nonlinear code are measured only after the transient associated with an initial perturbation has significantly decayed and the eigenmodes have fully settled. The excellent eigenmode agreement between the linear eigenvalue and nonlinear codes is demonstrated in Fig. 1.

\subsection{Nonlinear verification: comparison against kinetic simulations}

We have performed 2D nonlinear simulations with our algorithm (PIXIE2D code) to understand fast reconnection in low- $\beta$ regimes $[18,19]$. As part of this effort, we have compared against fully kinetic simulations (performed with the VPIC code [39]) in the large-guide-field regime to understand fundamental differences between kinetic and fluid reconnection in both $d_{e}>\rho_{s}$ and $d_{e}<\rho_{s}$ regimes [19]. For these simulations, we have used a Harris sheet equilibrium given by

$$
\Psi_{0}=-\lambda B_{0} \ln \left[\cosh \left(\frac{y}{\lambda}\right)\right]
$$

with $\lambda=1 / 25.64$ and $B_{0}$ is the asymptotic reconnecting field. The fluid simulations are run in a half-domain $x \in[-0.5,0.5], y \in[0,0.5]$. The boundary conditions are periodic in $x$, perfectly conducting at $y=0.5$, and symmetry/anti-symmetry at $y=0$. The number of mesh points is $1024 \times 512$. In addition, to resolve the dissipation scale, we pack the mesh along the $y$-axis by a factor of 8 to give a resolution of $1.22 \times 10^{-4}$ at the rational surface $(y=0)$. As in the linear verification section, we consider two different scenarios: electron-inertia-dominated $\left(d_{e}=0.01, \rho_{s}=0.002\right)$ and sound-radius-dominated $\left(d_{e}=0.002, \rho_{s}=0.01\right)$. For all cases, $\eta_{H}=10^{-9}$ and $\mu=10^{-5}$.

The VPIC simulations were performed in the full domain $x \in[-0.5,0.5], y \in[-0.5,0.5]$ with the same $\rho_{s}$ and $d_{e}$ as the fluid simulations. The PIC simulations were initialized with constant density and temperatures, and with force-balance provided by non-uniformity in the out-of-plane field (a force-free fluid equilibrium, but not an exact Vlasov one). Additional VPIC specific parameters for the electron-inertia-dominated case are: a guide-field-to-reconnecting-field ratio of $B_{g} / B_{0}=17.4$, a mass-ratio of $m_{i} / m_{e}=15.2$, ion-electron temperature ratio $T_{i} / T_{e}=0.25$, electron-plasma-frequency-to-cyclotron-frequency ratio $\omega_{p e} / \Omega_{c e}=2.5$, an equilibrium current sheet thickness equal to the ion-skin depth $\lambda / d_{i}=1$, and electron-thermal-velocity-tospeed-of-light ratio $v_{T e} / c=0.25$. For the sound-radius-dominated case, the VPIC parameters are $m_{i} / m_{e}=$ 

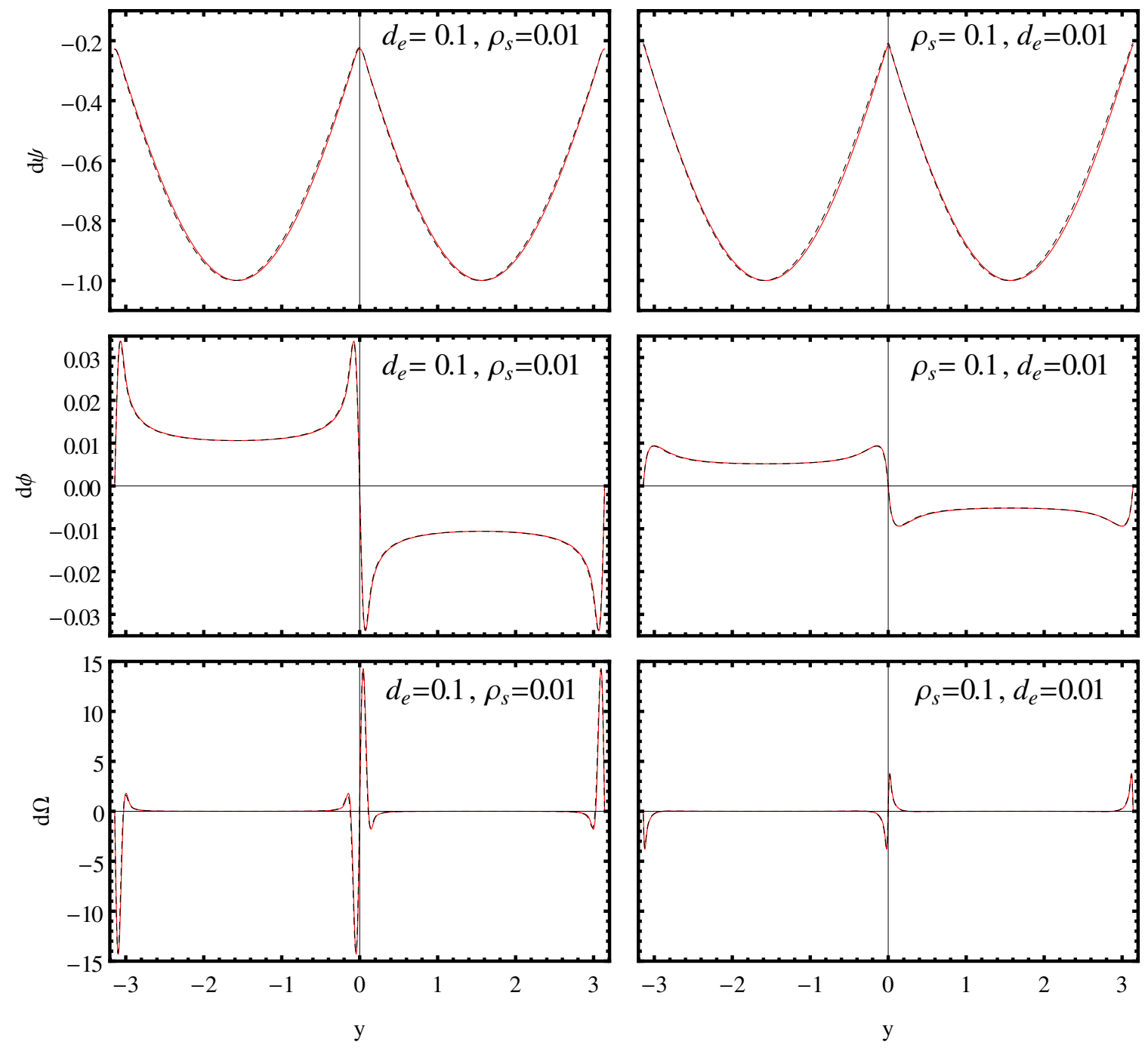

Figure 1: Linear eigenmode structure for the DPTM problem described in the text. Left panels depict eigenmode for the electroninertia-dominated case from both the eigenvalue code (solid red line) and our implementation (dashed black line). Right panels depict same quantities for the sound-radius-dominated case. 

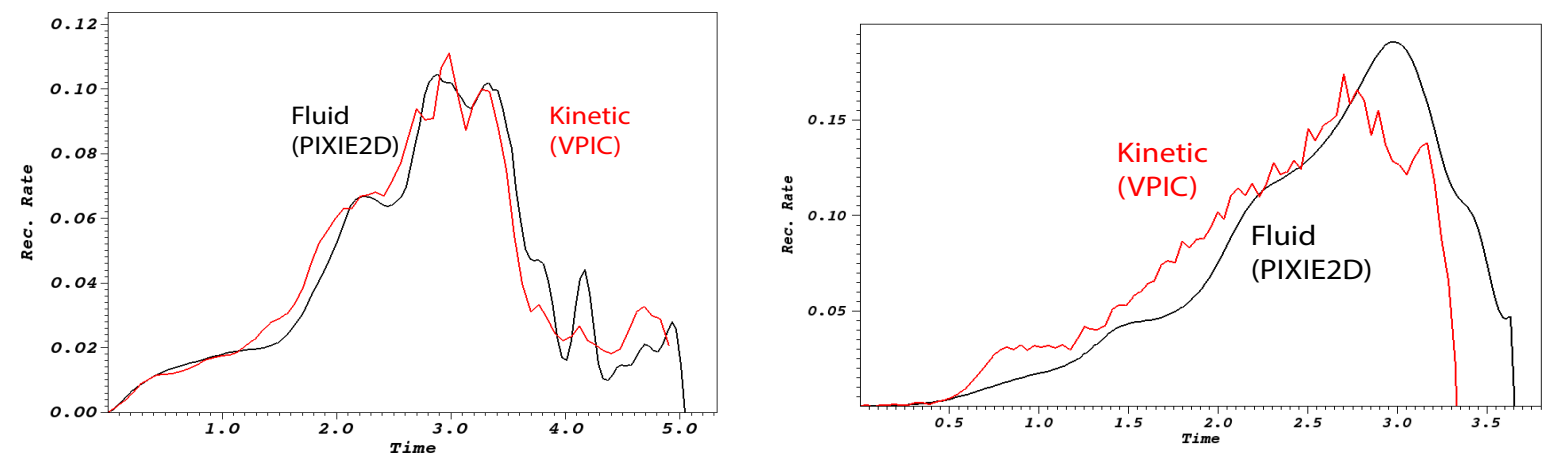

Figure 2: Comparison of time history of the reconnection rate between the two-field model (PIXIE2D) and fully kinetic (VPIC) simulations for the Harris sheet equilibrium in the electron-inertia-dominated regime (left) and the sound-radius-dominated regime (right).
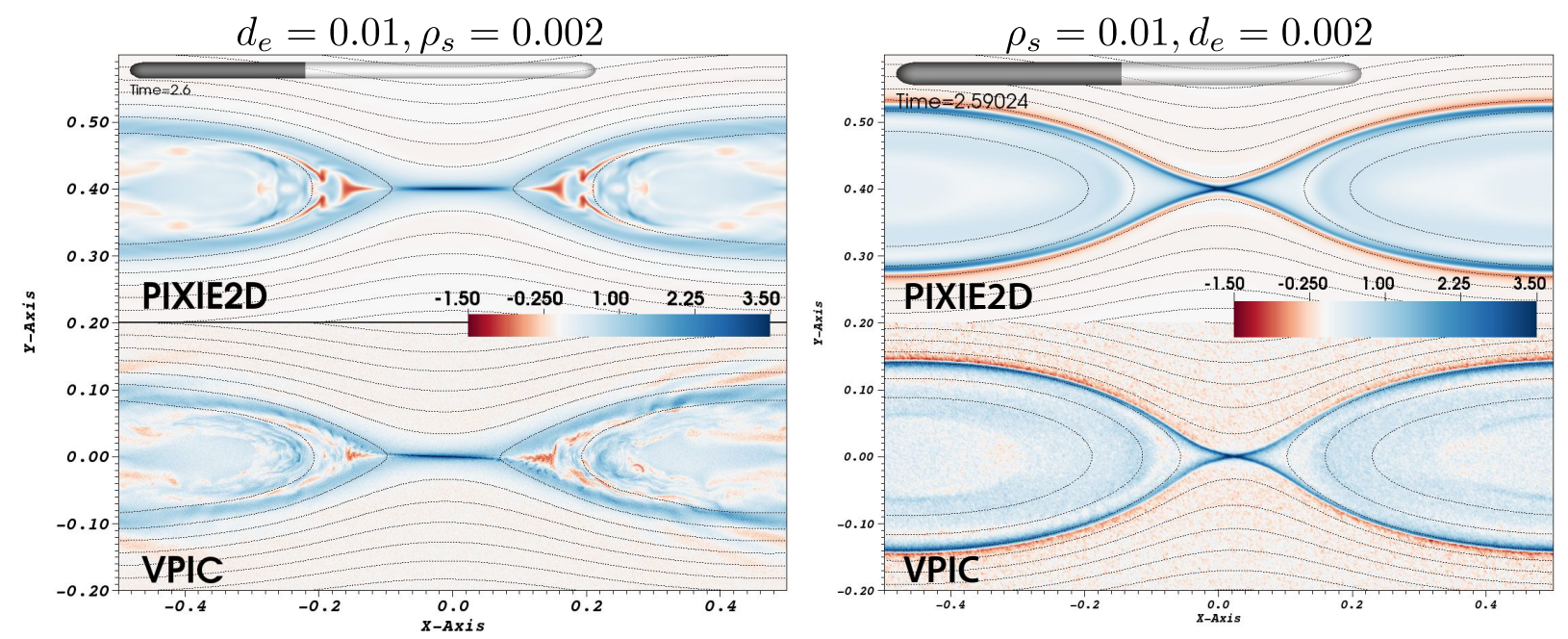

Figure 3: Snapshots of the current density (color scale) and magnetic flux (contours) from the PIXIE2D and VPIC simulations at $t=2.6$ (well into the nonlinear regime). The current density is normalized to the initial value at the center of the sheet. Movies comparing the full evolution of the system between fluid and kinetic regimes can be found in the supplementary material.

380.3, $B_{g} / B_{0}=12, v_{T e} / c=0.308, \omega_{p e} / \Omega_{c e}=10$, and $T_{i} / T_{e}=0.1$. The reconnection rates presented here are normalized to the Alfvénic rate at $4 \times \max \left[\rho_{s}, d_{e}\right]$ upstream of the dominant x-point, and for the VPIC runs we apply a mild relativistic correction to the Alfvén speed [40].

Figure 2 depicts the comparison of the fluid vs. kinetic time history of the reconnection rate in both $d_{e}>\rho_{s}$ and $\rho_{s}>d_{e}$ regimes, demonstrating excellent agreement in both magnitude and timing [19]. A snapshots of these runs from both fluid (PIXIE2D) and kinetic simulations (VPIC), showing the current density and magnetic flux at time $t=2.6$, is depicted in Figure 3. In addition, movies in the supplementary material show a comparison of the full evolution of these two simulations. It is clear that the fluid model reproduces the evolution of the current layer and flux surfaces from the kinetic simulations in this strongly non-linear regime, apart from small differences in electron-scale physics where the fluid and kinetic models are expected to differ. 
Table 1: Grid convergence study in the fast dispersive wave regime $\left(\rho_{s}=0.1, d_{e}=0.01\right)$ for the DPTM problem. In the Table, WCT stands for wall clock time. Results are averaged over 100 time steps with $\Delta t=1.0 . \Delta t_{\text {exp }}$ is the explicit timestep needed for numerical stability.

\begin{tabular}{ccccccc}
\hline Grid & $\eta_{H}$ & GMRES/ $\Delta t$ & Newton/ $\Delta t$ & WCT $(\mathrm{s})$ & $\mathrm{WCT}_{\exp } / \mathrm{WCT}$ & $\Delta t / \Delta t_{\exp }$ \\
\hline $32 \times 32$ & $3 \times 10^{-4}$ & 0.0 & 2.0 & 48 & 1.5 & 10 \\
$64 \times 64$ & $7.7 \times 10^{-5}$ & 0.0 & 2.0 & 192 & 9.4 & 41.5 \\
$128 \times 128$ & $1.9 \times 10^{-5}$ & 0.2 & 2.4 & 911 & 32 & 166 \\
$256 \times 256$ & $4.8 \times 10^{-6}$ & 0.4 & 2.6 & 4280 & 107 & 664 \\
$512 \times 512$ & $1.2 \times 10^{-6}$ & 1.4 & 2.9 & 21170 & 392 & 2656 \\
\hline
\end{tabular}

Table 2: Timestep convergence study for a $64 \times 64$ mesh for the DPTM problem with $\rho_{s}=0.1, d_{e}=0.01$. Results are averaged over a simulation time $T_{\max }=100$.

\begin{tabular}{cccccc}
\hline$\Delta t$ & GMRES/ $/ \Delta t$ & Newton/ $\Delta t$ & WCT (s) & WCT $_{\exp } / \mathrm{WCT}$ & $\Delta t / \Delta t_{\exp }$ \\
\hline 0.1 & 0.0 & 1.0 & 1100 & 1.6 & 4 \\
0.5 & 0.0 & 2.0 & 366 & 5 & 21 \\
1.0 & 0.0 & 2.0 & 192 & 9.4 & 41.5 \\
5 & 1.2 & 4.2 & 92 & 20 & 207
\end{tabular}

\subsection{Serial algorithmic performance}

We have performed both a grid convergence study and a time-step convergence study using the DPTM equilibrium in Sec. 5.1. We have focused in the numerically more challenging $\rho_{s}>d_{e}$ regime, due to the presence of fast dispersive waves (Sec. 2). In particular, we choose $\rho_{s}=0.1, d_{e}=0.01$. We employ uniform meshes for all computations in this section, to avoid difficulties introduced by packed meshes. Serial timings have been obtained in a MacBook Pro with a $2.8 \mathrm{GHz}$ Intel Core i7 processor running Mac OS X 10.10.

The results of the grid convergence study are presented in Table 1. Nonlinear solver performance data have been averaged over 100 time steps. In the preconditioner, we have used 2 semi-implicit iterations (i.e., $m=2$ in Eqs. 14, 15, and 16). We have fixed the implicit time step to $\Delta t=1.0$. We have adjusted the hyperresistivity with the grid resolution as $\eta_{H} \propto \rho_{s} v_{A} h^{2}$, with $h=\left(\frac{1}{\Delta x^{2}}+\frac{1}{\Delta y^{2}}\right)^{-1 / 2}$ [31], to provide dissipation at grid scale, and to prevent the numerical stiffness from the fourth-order operator to increase with mesh refinement (thus avoiding artificially decreasing the explicit time-step due to the Courant stability condition of the fourth-order dissipation operator). The Table shows the total number of GMRES iterations per time step, the total number of Newton iterations per time step, the wall-clock time (WCT) in seconds, the explicit-to-implicit WCT time ratio (the explicit WCT is estimated from 100 explicit time steps by multiplying the resulting WCT by $\Delta t / 100 \Delta t_{\text {exp }}$ ), and the implicit-to-explicit timestep ratio. The results in the Table demonstrate that the linear and nonlinear performance does not degrade under grid refinement despite the fact that the problem is becoming significantly more stiff as the mesh is refined (evidenced by the increase in the $\Delta t / \Delta t_{\text {exp }}$ ratio, which is about 2630 for the finest mesh considered). The number of GMRES iterations per time step averages to less than unity in some cases because the preconditioner is used to provide an initial condition to the GMRES iteration, which is often good enough to meet the relative GMRES convergence tolerance. The WCT speedup of the implicit solver vs. the explicit one scales with the total number of degrees of freedom $N$ (the implicit solver computational complexity scales optimally as $N$, whereas the explicit solver scales as $N^{2}$ due to the timestep stability limits associated with dispersive waves and/or dissipative terms). For the finest mesh considered, the WCT speedup is more than two orders of magnitude, demonstrating the efficiency of the approach.

We have also performed a time-step convergence study for the DPTM problem. The convergence results are averaged over a simulation time $T_{\max }=100$, and are presented in Table 2 for a $64 \times 64$ mesh. These 
Table 3: Parallel weak-scaling performance for DPTM problem with $\Delta t=1, \rho_{s}=0.1$, and $d_{e}=0.01$. The hyperbolic time step, $\Delta t_{\text {wave }}$, is the explicit stability time step when $\eta_{H}=\eta=\mu=0$, while $\Delta t_{\exp }$ is the explicit stability time step for actual values of the dissipation coefficients. Results are averaged over 10 time steps.

\begin{tabular}{c|ccccccccc}
$N_{p}$ & Grid & $\eta_{H}$ & GMRES $/ \Delta t$ & Newt/ $/ \Delta t$ & WCT $(\mathrm{s})$ & WCT/PC & $\frac{\mathrm{WCT}_{\text {exp }}}{\text { WCT }}$ & $\frac{\Delta t}{\Delta t_{\text {exp }}}$ & $\frac{\Delta t}{\Delta t_{\text {wave }}}$ \\
\hline 1 & $64 \times 64$ & $7.7 \times 10^{-5}$ & 0 & 1.9 & 50.3 & 26.5 & 8.5 & 41.5 & 27.1 \\
4 & $128 \times 128$ & $1.9 \times 10^{-5}$ & 0 & 1.9 & 53.7 & 28.3 & 33.3 & 166 & 77.8 \\
16 & $256 \times 256$ & $4.8 \times 10^{-6}$ & 0 & 1.9 & 59.7 & 31.4 & 131.5 & 664 & 215.7 \\
64 & $512 \times 512$ & $1.2 \times 10^{-6}$ & 0 & 1.9 & 74.9 & 39.4 & 504.6 & 2656 & 555.6 \\
256 & $1024 \times 1024$ & $3.0 \times 10^{-7}$ & 0 & 1.9 & 83.5 & 43.9 & 1946.5 & 10627 & 1315.2 \\
1024 & $2048 \times 2048$ & $7.5 \times 10^{-8}$ & 0 & 1.9 & 89 & 46.8 & 8064.4 & 42508 & 2908.5 \\
4096 & $4096 \times 4096$ & $1.9 \times 10^{-8}$ & 3.6 & 3.6 & 346 & 48.1 & 10127.8 & 170030 & 6148.5
\end{tabular}

results demonstrate that performance is a weak function of the implicit time step, and that the algorithm performs well even for really large time steps (several Alfvén times).

\subsection{Parallel weak-scaling performance}

We have performed a weak-scaling parallel performance test with the DPTM test case, using the same simulation parameters as with the serial case, except now we are averaging over 10 time steps instead of 100. In particular, we also keep the implicit timestep constant, $\Delta t=1$, which results in increased numerical stiffness with grid refinement (and therefore with processor count). Runs have been performed in the Mustang HPC Linux Cluster at LANL. Mustang is an Appro Xtreme-X cluster with dual-socket AMD 12core MagnyCours processors. The cluster interconnect is comprised of a Mellanox InfiniScale IV 4x-QDR interconnect configured in a fully non-blocking Full Bisectional Bandwidth (FBB) fat-tree topology.

The results are given in Table 3, which shows, in addition to the quantities in previous Tables, the WCT per preconditioning call (WCT/PC, which is used as a measure of the parallel scalability of our solver), and timestep ratios with respect to both hyperbolic $\Delta t_{\text {wave }}$ and explicit $\Delta t_{\text {exp }}$ timesteps (the former is a measure of hyperbolic stiffness only, whereas the latter is a measure of total numerical stiffness, including dissipation). The number of preconditioning calls per time step, PC, for our right-preconditioned FGMRES implementation is given by PC $=$ Newton + GMRES. The first number counts the number of preconditioning calls to find the initial guess per Jacobian solve, and the second number counts the preconditioner call per GMRES iteration; note that FGMRES does not need a final preconditioning call to reconstruct the solution [36].

The parallel results in Table 3 are an objective measure of the overall cost of our algorithm to reach a given final simulation time, and accounts for both algorithmic scalability (i.e., iteration count variation with grid refinement) and parallel scalability of our solver implementation. Algorithmic scalability is illustrated by the numbers in the GMRES/ $\Delta t$ and Newton/ $\Delta t$ columns. Parallel scalability of our solver is illustrated by the WCT/PC column. These results demonstrate that our implementation scales well in parallel, with the WCT/PC figure of merit increasing less than a factor of two between 1 and 4096 cores. Our solver also scales well algorithmically, with convergence rates degrading only for the largest mesh size considered, when $\Delta t / \Delta t_{\text {exp }} \sim 2 \times 10^{5}$. As a result, to reach a given final simulation time, the total WCT increases by a factor of 7 between the single-core run and the 4096-core run. To put this result in perspective, for these same conditions a perfectly parallelized explicit algorithm would have resulted in a WCT increase of 4096, owing to timestep stability constraints restricting the time step as $\Delta t \sim \Delta x^{2}$ (either due to diffusion or dispersive waves). The actual explicit-to-implicit WCT ratio is about $10^{4}$ for the finest mesh considered in Table 3.

For the conditions in Table 3, it is apparent that the explicit timestep is dominated by the diffusion Courant condition $\left(\Delta t_{\text {exp }} \ll \Delta t_{\text {wave }}\right)$, and in particular by the hyperresistivity. This is so because the fast dispersive waves are tempered by the electron skin depth $d_{e}$ (Eq. 5). In order to test the sensitivity of our 
Table 4: Parallel weak-scaling performance for DPTM problem with $\Delta t=1, \rho_{s}=0.1$, and $d_{e}=0$. Results are averaged over 10 time steps.

\begin{tabular}{c|cccccccc}
$N_{p}$ & Grid & $\eta_{H}$ & GMRES $/ \Delta t$ & Newt $/ \Delta t$ & WCT $(\mathrm{s})$ & WCT/PC & $\frac{\Delta t}{\Delta t_{\text {exp }}}$ & $\frac{\Delta t}{\Delta t_{\text {wave }}}$ \\
\hline 1 & $64 \times 64$ & $7.7 \times 10^{-5}$ & 0 & 1.9 & 50.6 & 26.6 & 41.5 & 33.3 \\
4 & $128 \times 128$ & $1.9 \times 10^{-5}$ & 0 & 1.9 & 53.6 & 28.2 & 166.0 & 113.2 \\
16 & $256 \times 256$ & $4.8 \times 10^{-6}$ & 0 & 1.9 & 59.7 & 31.4 & 664.2 & 412.3 \\
64 & $512 \times 512$ & $1.2 \times 10^{-6}$ & 0 & 1.9 & 77.0 & 40.5 & 2656.7 & 1567.8 \\
256 & $1024 \times 1024$ & $3.0 \times 10^{-7}$ & 0 & 1.9 & 84.1 & 44.3 & 10627 & 6108.0 \\
1024 & $2048 \times 2048$ & $7.5 \times 10^{-8}$ & 0 & 1.9 & 93.7 & 49.3 & 42508 & 24106.8 \\
4096 & $4096 \times 4096$ & $1.9 \times 10^{-8}$ & 3.8 & 3.6 & 380.6 & 51.4 & 170030 & 95776.3
\end{tabular}

algorithm's performance to $d_{e}$, we have rerun the tests in the Table with $d_{e}=0$. In this case, $\Delta t_{\text {exp }} \sim \Delta t_{\text {wave }}$. The results are presented in Table 4, and show that the solver performance is essentially unchanged, demonstrating the insensitivity of the performance of the solver to $d_{e}$, and the ability of our implicit algorithm to deal with arbitrarily large hyperbolic stiffness. WCT speedups vs. the explicit algorithm for the $d_{e}=0$ case (not shown) are comparable to those in Table 3.

\section{Conclusions}

We have demonstrated a correct, efficient, and algorithmically scalable fully implicit solver for the low- $\beta$ extended MHD model. The solver employs Jacobian-free Newton-Krylov methods, accelerated by physics-based preconditioners for efficiency. The preconditioner formulation produces a single paraboliclike equation that can be efficiently inverted using classical multigrid methods. Grid and timestep convergence studies in the numerically challenging fast-dispersive-wave regime $\left(\rho_{s}>d_{e}\right)$ have demonstrated the excellent algorithmic performance of the approach, both in convergence rates and vs. explicit methods. In particular, serial speedups of $\sim 400$ vs. explicit methods have been demonstrated for a $512 \times 512$ mesh. Parallel speedups reach $10^{4}$ vs. explicit for a $4096 \times 4096$ mesh. This is to be compared to the (at most) single-order-of-magnitude speedups demonstrated in earlier studies for comparable-size problems [27]. A parallel weak-scaling study with up to $N_{p}=4096$ cores (which is the maximum number of cores available to us for this study) shows that the wall clock time scales very weakly with $N_{p}$ from $N_{p}=1$ to 4096 . Linear and nonlinear verification studies have shown the model is solved correctly. In particular, we have performed linear-theory comparisons against a linear eigenvalue code in both the electron-inertia-dominated $\left(d_{e}>\rho_{s}\right)$ and sound-radius-dominated $\left(d_{e}<\rho_{s}\right)$ regimes, demonstrating excellent agreement in both eigenvalues and eigenfunctions. Nonlinear comparisons of the two-field low- $\beta$ extended MHD algorithm against first-principles kinetic simulations for a Harris sheet in similar $d_{e}$ vs. $\rho_{s}$ regimes have demonstrated remarkable nonlinear agreement.

\section{Acknowledgments}

We acknowledge useful discussions with W. Daughton and A. Simakov. We would like to thank W. Daughton for his help in setting up and using the VPIC code. This research used resources provided by the Los Alamos National Laboratory Institutional Computing Program, which is supported by the U.S. Department of Energy National Nuclear Security Administration under Contract No. DE-AC52-06NA25396. The work was funded by the DOE Offices of Fusion Energy Sciences and Applied Scientific Computing Research.

[1] R. D. Hazeltine, M. Kotschenreuther, and P. J. Morrison, "A four-field model for tokamak plasma dynamics," Phys. Fluids, vol. 28, no. 8, pp. 2466-2477, 1985. 
[2] T. J. Schep, F. Pegoraro, and B. N. Kuvshinov, "Generalized two-fluid theory of nonlinear magnetic structures,” Phys. Plasmas, vol. 1, no. 9, pp. 2843-2852, 1994.

[3] D. Grasso, F. Pegoraro, F. Porcelli, and F. Califano, "Hamiltonian magnetic reconnection," Plasma Phys. Control. Fusion, vol. 41, no. 12, pp. 1497 - 515, 1999.

[4] S. I. Braginskii, "Transport processes in a plasma," in Reviews of Plasma Physics (M. A. Leontovich, ed.), vol. 1, pp. 205-311, New York: Consultants Bureau, 1965.

[5] M. Yamada, F. M. Levinton, N. Pomphrey, R. Budny, J. Manickam, and Y. Nagayama, "Investigation of magnetic reconnection during a sawtooth crash in a high-temperature tokamak plasma," Phys. Plasmas, vol. 1, p. 3269, 1994.

[6] I. T. Chapman, R. Scannell, W. A. Cooper, J. P. Graves, R. J. Hastie, G. Naylor, , and A. Zocco, "Magnetic reconnection triggering magnetohydrodynamic instabilities during a sawtooth crash in a tokamak plasma," Phys. Rev. Lett., vol. 105, p. 255002, 2010.

[7] Y. Ono, H. Tanabe, Y. Hayashi, T. Ii, Y. Narushima, T. Yamada, M. Inomoto, , and C. Z. Cheng, "Ion and electron heating characteristics of magnetic reconnection in a two flux loop merging experiment," Phys. Rev. Lett., vol. 107, p. 185001, 2011.

[8] A. Stanier, P. Browning, M. Gordovskyy, K. G. Mc-Clements, M. P. Gryaznevich, and V. S. Lukin, "Two-fluid simulations of driven reconnection in the mega-ampere spherical tokamak," Phys. Plasmas, vol. 20, p. 122302, 2013.

[9] E. Priest and T. Forbes, Magnetic reconnection: MHD theory and application. Cambridge, UK: Cambridge University Press, 2000.

[10] A. Y. Aydemir, "Nonlinear studies of $\mathrm{m}=1$ modes in high-temperature plasmas," Phys. Fluids B, vol. 4, p. $3469,1992$.

[11] M. Ottaviani and F. Porcelli, "Nonlinear collisionless magnetic reconnection," Phys. Rev. Lett., vol. 71, p. $3802,1993$.

[12] M. Ottaviani and F. Porcelli, "Fast nonlinear magnetic reconnection," Phys. Plasmas, vol. 2, p. 4104, 1995.

[13] E. Cafaro, D. Grasso, F. Pegoraro, F. Porcelli, and A. Saluzzi, "Invariants and geometric structures in nonlinear hamiltonian magnetic reconnection,” Phys. Rev. Lett., vol. 80, no. 20, pp. 4430 - 3, 1998.

[14] R. Fitzpatrick, "Scaling of forced magnetic reconnection in the hall-magnetohydrodynamical taylor problem with arbitrary guide field," Phys. Plasmas, vol. 11, no. 8, p. 3961, 2004.

[15] A. Bhattacharjee, K. Germaschewski, and C. S. Ng, "Current singularities: Drivers of impulsive reconnection," Phys. Plasmas, vol. 12, p. 042305, 2005.

[16] S. Schmidt, S. Günter, , and K. Lackner, "Reconnection in semicollisional, low-beta plasmas," Phys. Plasmas, vol. 16, p. 072302, 2009.

[17] A. N. Simakov, L. Chacón, and A. Zocco, "Fundamental role of ion viscosity on fast magnetic reconnection in large-guide-field regimes," Phys. Plasmas, vol. 17, p. 060701, 2010.

[18] A. Stanier, A. Simakov, L. Chacón, and W. Daughton, "Fast magnetic reconnection with large guide fields," Phys. Plasmas, vol. 22, p. 010701, 2015. 
[19] A. Stanier, A. Simakov, L. Chacón, and W. Daughton, "Fluid vs. kinetic magnetic reconnection with strong guide fields," Phys. Plasmas, vol. 22, p. 101203, 2015.

[20] M. Hirota, Y. Hattori, and P. J. Morrison, "Explosive magnetic reconnection caused by an X-shaped current-vortex layer in a collisionless plasma," Physics of Plasmas, vol. 22, no. 5, 2015.

[21] B. N. Rogers, R. E. Denton, J. F. Drake, and M. A. Shay, "Role of dispersive waves in collisionless magnetic reconnection.," Phys. Rev. Lett., vol. 87, no. 19, p. 195004, 2001.

[22] J. Birn, J. F. Drake, M. A. Shay, B. N. Rogers, R. E. Denton, M. Hesse, M. M. Kuznetsova, Z. W. Ma, A. Bhattacharjee, A. Otto, and P. L. Pritchett, "Geospace Environment Modeling (GEM) magnetic reconnection challenge," J. Geophys. Res., vol. 106, no. A3, pp. 3715-3719, 2001.

[23] Z. W. Ma and A. Bhattacharjee, "Hall magnetohydrodynamic reconnection: The Geospace Environment Modeling challenge," J. Geophys. Res., vol. 106, no. A3, pp. 3773-3782, 2001.

[24] D. Del Sarto, F. Califano, and F. Pegoraro, "Secondary instabilities and vortex formation in collisionless-fluid magnetic reconnection,” Phys. Rev. Lett., vol. 91, no. 23, p. 235001, 2003.

[25] D. Grasso, D. Borgogno, and F. Pegoraro, "Secondary instabilities in two-and three-dimensional magnetic reconnection in fusion relevant plasmasa)," Phys. Plasmas, vol. 14, no. 5, p. 055703, 2007.

[26] K. Germaschewski, A. Bhattacharjee, and C.-S. Ng, "The magnetic reconnection code: an amr-based fully implicit simulation suite," in Numerical Modeling of Space Plasma Flows (N. B. Pogorelov and G. P. Zank, eds.), vol. 359 of ASP Conference Series, 2006.

[27] S. Ovtchinnikov, F. Dobrian, X.-C. Cai, and D. Keyes, "Additive schwarz-based fully coupled implicit methods for resistive hall magnetohydrodynamic problems," J. Comput. Phys., vol. 225, pp. 1919 1936, 2007.

[28] M. Kraus, E. Tassi, and D. Grasso, "Variational integrators for reduced magnetohydrodynamics," $J$. Comput. Phys., vol. 321, pp. 435 - 458, 2016.

[29] L. Chacón, D. A. Knoll, and J. M. Finn, "Implicit, nonlinear reduced resistive MHD nonlinear solver," J. Comput. Phys., vol. 178, no. 1, pp. 15-36, 2002.

[30] L. Chacón, "An optimal, parallel, fully implicit Newton-Krylov solver for three-dimensional viscoresistive magnetohydrodynamics," Phys. Plasmas, vol. 15, p. 056103, 2008.

[31] L. Chacón and D. A. Knoll, "A 2D high- $\beta$ Hall MHD implicit nonlinear solver," J. Comput. Phys., vol. 188, no. 2, pp. 573-592, 2003.

[32] L. Chacón, "Scalable solvers for 3D magnetohydrodynamics," J. Physics: Conf. Series, vol. 125, p. 012041, 2008.

[33] G. D. Byrne and A. C. Hindmarsh, "A polyalgorithm for the numerical solution of ordinary differential equations," ACM Transactions on Mathematical Software, vol. 1, no. 1, pp. 71-96, 1975.

[34] C. T. Kelley, Iterative Methods for Linear and Nonlinear Equations. Philadelphia: SIAM, 1995.

[35] R. S. Dembo, S. C. Eisenstat, and T. Steihaug, "Inexact Newton methods," SIAM J. Numer. Anal., vol. 19, no. 2, p. 400, 1982. 
[36] Y. Saad, "A flexible inner-outer preconditioned GMRES algorithm," SIAM J. Sci. Comput., vol. 14, no. 2, pp. 461-469, 1992.

[37] W. L. Briggs, A Multigrid Tutorial. Philadelphia, PA: SIAM, 1987.

[38] P. Wesseling, An Introduction to Multigrid Methods. Chichester: John Wiley \& Sons, 1992.

[39] K. J. Bowers, B. J. Albright, L. Yin, B. Bergen, and T. J. T. Kwan, "Ultrahigh performance threedimensional electromagnetic relativistic kinetic plasma simulation,” Phys. Plasmas, vol. 15, no. 5, p. 055703, 2008.

[40] Y.-H. Liu, W. Daughton, H. Karimabadi, H. Li, and S. Peter Gary, "Do dispersive waves play a role in collisionless magnetic reconnection?," Phys. Plasmas, vol. 21, no. 2, p. $022113,2014$. 